\title{
Spectroscopy on triphenylamine and its van der Waals complexes
}

\author{
Gerard Meijer, Giel Berden, W. Leo Meerts \\ Depariment of Molecular and Laser Physics, Cntversity of Nlymegen, Toernoolveld, 6525 ED Nimegen, The Netherlands \\ Heinrich E. Hunziker, Mattanjah S. de Vries and H. Russell Wendt \\ IBM Almaden Research Center, 650 Harry Road, San José CA 95120, USA
}

Received 14 November 1991

\begin{abstract}
Both vibrationally and rotationally resolved spectra of the $S_{1} \leftarrow S_{0}$ transition in jet-cooled triphenylamine (TPA) around 340$320 \mathrm{~nm}$ are reported. Medium resolution spectra $\left(0.5-1.0 \mathrm{~cm}^{-1}\right.$ resolution) are recorded using $(1+1)$-resonance enhanced multi photon ionization (REMPI) with mass selective time-of-flight (TOF) detection in a pulsed molecular beam apparatus. The origin of the $S_{1} \leftarrow S_{0}$ transition is at $29520.7 \mathrm{~cm}^{-1}$, higher than halfway to the conızation potential (IP) found at $6.89 \mathrm{eV}$. A vibrational progression in the symmetric torsion mode $\left(114 \mathrm{~cm}^{-1}\right)$ as well as in the symmetric $\mathrm{C}-\mathrm{N}$ stretching mode $\left(280 \mathrm{~cm}^{-1}\right)$ is observed in the electronic spectra. The spectrum of the most abundant isomer of the TPA-Ar (TPA-Kr) complexes is blue-shifted by 211 $\mathrm{cm}^{-1}\left(216 \mathrm{~cm}^{-1}\right)$ with respect to the spectrum of the free TPA molecule High-resolution (the resolution mainly being determined by the natural linewidth of the transition, 1.e. $36 \mathrm{MHz}$ ) spectra are recorded using laser induced fluorescence (LIF) in a $\mathrm{cw}$ molecular beam apparatus. Individual rotational transitions are resolved and the spectrum shows unambiguously that TPA is a symmetric top molecule. The rotational constant $B^{\prime \prime}$ in the $\mathrm{S}_{0}$ state of TPA is equal to $B^{\prime \prime}=403.7 \pm 0.5 \mathrm{MHz}$. Upon $\mathrm{S}_{1} \leftarrow \mathrm{S}_{0}$ excitation both $B$ and $C$ increase with $7.4 \pm 0.1 \mathrm{MHz}$ and $2.8 \pm 0.1 \mathrm{MHz}$, respectively. The spectrum of the blue-shifted TPA-Ar isomer is the spectrum of a symmetric top molecule as well, and therefore the Ar atom has to be located on the $\mathrm{C}_{3}$ symmetry axis, either on top of or underneath the umbrella formed by the phenyl rings. It appears that when Ar or Kr forms a complex with TPA, the first Ar, $K_{r}$, atom goes preferentially in a position on the $C_{3}$ symmetry axis of TPA, a position which causes an abnormal blucshift of the spectrum. With the first rare-gas atom located in this special position, the second rare-gas atom is forced into a "normal" position, 1.e. above one of the phenyl rings, causing a normal red-shift with respect to the TPA-Ar complex.
\end{abstract}

\section{Introduction}

A spectroscopic investigation of triphenylamine (TPA) is of special interest for the understanding of the interaction between the lone pair electron of the $\mathrm{N}$ atom and the $\pi$ electrons of the adjoining aromatic phenyl groups ( $n \pi$ conjugation). The interplay betwecn $n \pi$ conjugation and steric hindrance becomes more important in going from aniline via diphenylamine to triphenylamine; the character of the lone pair electron in TPA will be strongly influenced by the molecular conformation. Up to now several experiments have been performed to determine the geometrical structure of TPA in the gas phase [1], in the liquid phase [2-5], as wcll as in solid films [6], but a definite structure for TPA has not yet emerged from these measurements. The geometrical structure of the TPA cation in the gas phase and in solid films has also only been determined indirectly via a comparison of measured and calculated photoelectron spectra [7].

In the present work both vibrationally and rotationally resolved spectra of jet-cooled TPA are reported. The geometrical structure of TPA in the $S_{0}$ and in the $S_{1}$ state is deduced from the measured rotational constants.

Molecular beam techniques have been used extensively to study van der Waals complexes consisting of organic aromatic hydrocarbons bonded to rare-gas atoms. Such heteroclusters have provided an interdisciplinary testing ground for the energetics and the dynamics related to intermolecular potential, cluster growth and solvent perturbation [8,9]. The microscopic solvent shifts of these heteroclusters are generally to lower energy, are linearly dependent on the polarizability of the rare-gas atom and are additive 
for rare-gas atoms in equivalent structural positions. Different structural isomers have different spectral shifts. A solvent shift towards higher energy has been reported for fluorobenzene [10], phenyl acetylene [11], paraxylene [12] and more recently for aniline [13,14]; the cluster of each of these molecules with three Ar atoms shows a blue-shift with respect to the cluster with two Ar atoms.

In the present work we report spectra of various isomers of the TPA-Ar, $\mathrm{Kr}$ vdW complexes. The spectra of the most abundant TPA-Ar, $\mathrm{Kr}$ isomers show a large anomalous blue-shift with respect to the spectrum of the free TPA molecule. The structure of these blue-shifted vdW complexes is determined, and the origin of the blue-shift is discussed.

\section{Vibrationally resolved (1+1)-REMPI spectroscopy}

\subsection{Experimental setup}

The search for the $S_{1} \leftarrow S_{0}$ transition in TPA was made using pulsed lasers and a pulsed molecular beam apparatus at the IBM Almaden Research Center. The vapour pressure of TPA at room temperature is about $10^{-5}$ Torr, and laser vaporization followed by supersonic cooling in a rare-gas expansion was used to reproduce an intense pulsed beam of jet-cooled TPA. The laser desorption jet-cooling apparatus has been described in detail recently [15], and only a short experimental description will be given here.

A KrF excimer laser (approximately $50 \mu \mathrm{J}$ in a 0.25 mm diameter spot) is used to desorb the TPA from a piece of fritted glass placed very close (within two nozzle diameters) to the orifice of a supersonic jet. The fritted glass acts as a matrix for the TPA and yields a steady supply of TPA to the surface. The supersonic jet is formed by expanding $8-10 \mathrm{~atm}$ of rare gas ( $\mathrm{Ar}$ or $\mathrm{Kr}$ ) through a $0.5 \mathrm{~mm}$ nozzle. The laser desorbed molecules are entrained in the jet and their internal degrees of freedom are cooled by multiple collisions in the expansion region. Rotational temperatures down to $5 \mathrm{~K}$ are obtained in this way. The jet-cooled desorbed molecules obtain the same velocity as the supersonically expanding rare gas and arrive in the detection chamber after having passed through a 1 by $4 \mathrm{~mm}$ slit skimmer.

In the detection chamber the laser desorbed TPA molecules are detected via resonant one photon $\mathrm{S}_{1} \leftarrow \mathrm{S}_{0}$ laser excitation followed by one photon ionization, either by the same or by a longer wavelength laser. Nd:YAG pumped pulsed dye lasers with a typical bandwidth of $0.4 \mathrm{~cm}^{-1}$ are used for excitation and ionization. The ions are formed between the extraction plates of a Wiley-McLaren type linear timeof-flight (TOF) mass spectrometer. A mass resolution $M / \Delta M$ of $300-400$ is obtained, enough to separate TPA (245 amu) from its ${ }^{13} \mathrm{C}$ containing isotope. Every single laser pulse yields a complete TOF massspectrum, which is displayed on a digital oscilloscope and stored in a PC. With the PC, gates are set over various mass ranges, and wavelength spectra of several molecules (or molecular fragments) can be measured simultaneously.

\subsection{The $S_{1} \leftarrow S_{0}$ transition of $T P A$}

The origin of the lowest allowed singlet transition in TPA is found around $339 \mathrm{~nm}$; the corresponding transitions in aniline and diphenylamine have their respective origins at 294 [16] and $308 \mathrm{~nm}$ [15]. In the upper part of fig. 1, the $(1+1)$-REMPI spectrum of jet-cooled TPA is shown. A tunable, pulsed dye laser with a fluence of $1.0 \mathrm{~mJ} / \mathrm{cm}^{2}$ is used to induce the $S_{1} \leftarrow S_{0}$ transition in TPA, and to subsequently ionize from the $S_{1}$ state. Positive ions of the parent mass (245 amu) are detected. Under these conditions, and even when two orders of magnitude higher laser fluences are used, no fragmentation of the TPA upon ionization is observed.

In the TPA spectrum shown in fig. 1a, two vibrational progressions are visible, corresponding to vibrational modes at 114 and $280 \mathrm{~cm}^{-1}$. Both of the progressions are very harmonic. These are the only two low-frequency modes that appear strongly when a low excitation fluence is used. Although not indicated in the figure, other slightly weaker fundamental modes can be recognized in the low-fluence excitation spectrum around $383,583,715$ and $742 \mathrm{~cm}^{-1}$. All these modes combine again with the 114 and 280 $\mathrm{cm}^{-1}$ vibrations.

When higher laser fluences are used for the resonant excitation step, a number of extra peaks appear. A $(1+1)$-REMPI spectrum of jet-cooled TPA recorded with a laser fluence of $100 \mathrm{~mJ} / \mathrm{cm}^{2}$ is shown in fig. 2 . The stronger peaks in the spectrum are all 

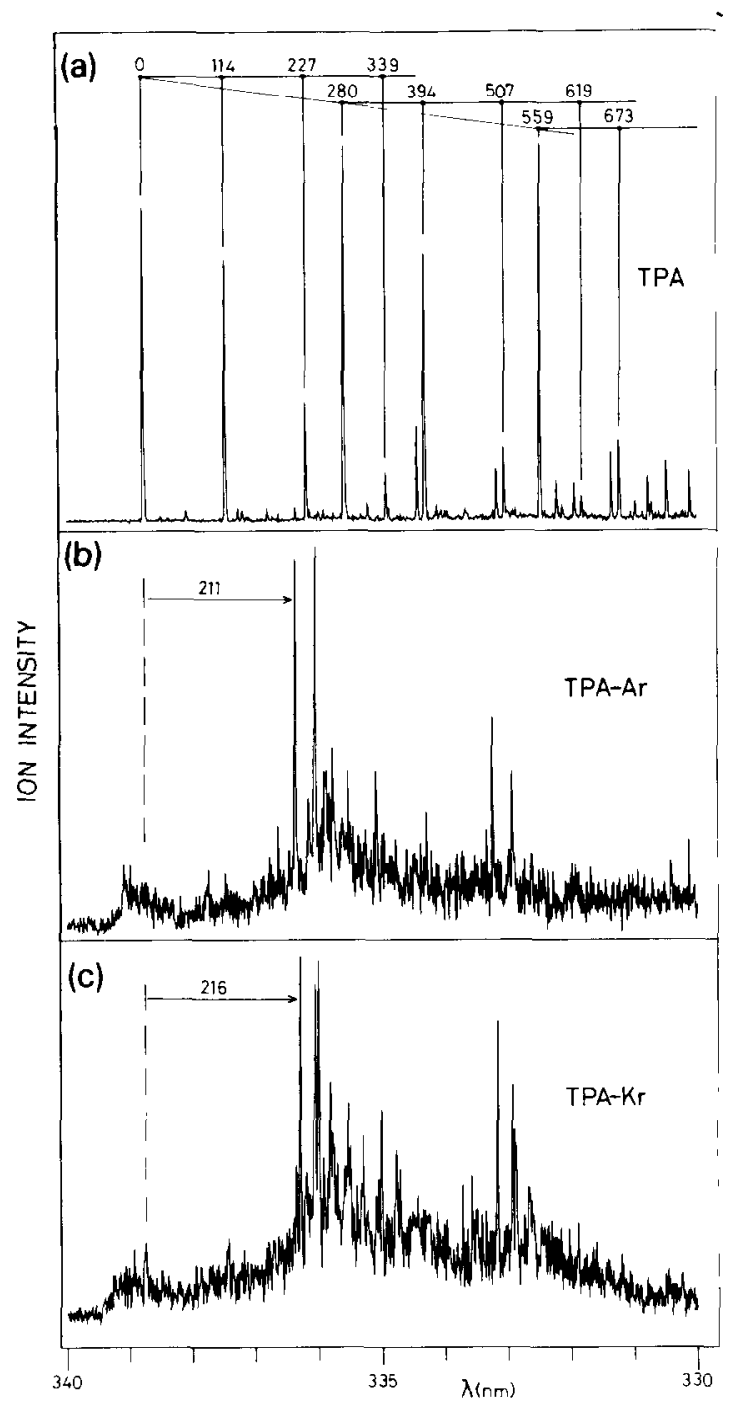

Fig. 1. Vibrationally resolved $(1+1)$-REMPI spectrum of jetcooled TPA (a), TPA-Ar (b) and TPA-Kr (c). In all cases the parent ion is mass selectively detected. In the spectrum of TPA, two vibrational progressions are indicated (values in $\mathrm{cm}^{-1}$ ). In the lower panels the shift of the van der Waals complexes relative to the free TPA is indicated (in $\mathrm{cm}^{-1}$ ).

saturated by the excitation laser (they should thus be equally intense) and clipped by the detection electronics to better show the weaker features. The peaks at 114 and $280 \mathrm{~cm}^{-1}$ are indicated again to aid in comparing this spectrum to the one shown in fig. 1a. $\Lambda t$ low frequency there are cxtra peaks at $59,131,137$ and at $143 \mathrm{~cm}^{-1}$. All these weaker peaks combine again with the 114 and $280 \mathrm{~cm}^{-1}$ fundamental. The two weak peaks indicated with an asterisk in fig. 1 show the same saturation behaviour as the strong fundamentals. These peaks have always approximately $1 \%$ of the intensity of the main peaks, also when low excitation fluences are used.Although these peaks are 23 and $32 \mathrm{~cm}^{-1}$ to the blue of the TPA origin, we believe them to be hot bands, originating from vibrationally excited TPA, as will be discussed in section 4.

In an experiment in which two frequency doubled pulsed dye lasers pumped with two independent $\mathrm{Nd}$ : YAG lasers were used, we measured the ionization potential (IP) of TPA. A low-fluence dye laser $\left(1.0 \mathrm{~mJ} / \mathrm{cm}^{2}\right)$ was used to resonantly excite TPA from the $S_{0}$ to the $S_{1}$ state, whereas a second dye laser with two orders of magnitude higher fluence was used to ionize TPA from the $S_{1}$ state. A wavelength scan of the second dye laser, showing the onset of ionization, is shown in fig. 3. In fig. 3a the onset of ionization is shown for jet-cooled TPA, resonantly excited to the vibrationless level in the electronically excited $S_{1}$ state. The onset of ionization is seen around 385 $\mathrm{nm}$, at $25968 \mathrm{~cm}^{-1}$ to be more precise. If TPA is resonantly excited to the $114 \mathrm{~cm}^{-1}$ vibration in the $S_{1}$ state, one would expect the onset of ionization to appear when the ionizing laser has an $114 \mathrm{~cm}^{-1}$ lower frequency, i.e. around $386.7 \mathrm{~nm}$. In fig. $3 \mathrm{~b}$ this spectrum is shown and there is only a $10 \mathrm{~cm}^{-1}$ red-shift of the strongest onset of the IP, however. This indicates that the Franck-Condon factors for the transition from the $S_{1}$ state of the neutral to the ground state of the positive ion are more or less diagonal for this mode; from the one quantum excited $114 \mathrm{~cm}^{-1}$ vibration in the TPA $S_{1}$ state preferentially TPA ground state ions that have one quantum excited in the corresponding vibration are produced. Moreover, it can be deduced from the spectrum in fig. 3b that the frequency of the vibration in the ion that corresponds to the vibration of $114 \mathrm{~cm}^{-1}$ in the neutral is approximately $10 \mathrm{~cm}^{-1}$ lower, i.e. around 104 $\mathrm{cm}^{-1}$. In fig. $3 \mathrm{c}$ something similar is seen when TPA is resonantly excited to the $280 \mathrm{~cm}^{-1}$ vibration in the $S_{1}$ state and the ionization laser is scanned over the ionization onset. Although now a weak onset of ionization is observed around $389 \mathrm{~nm}$, as expected, it can be concluded from the strong step in the ionization continuum around $385 \mathrm{~nm}$ that again preferen- 


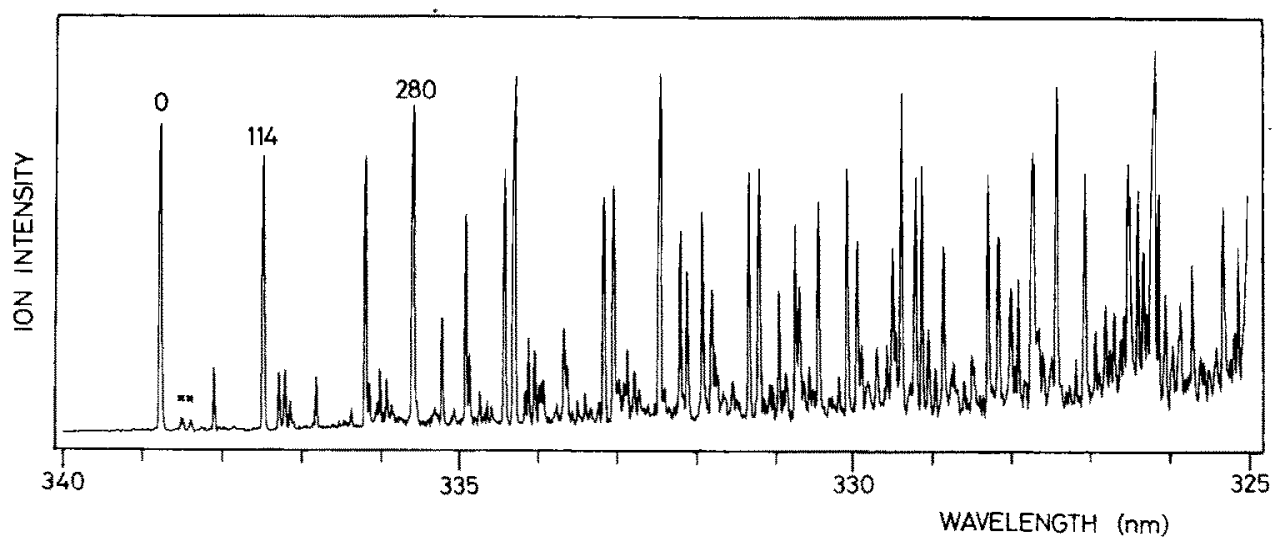

Fig. 2. Vibrationally resolved $(1+1)$-REMPI spectrum of jet-cooled TPA using $100 \mathrm{~mJ} / \mathrm{cm}^{2}$ for cxcitation and ionızation. The stronger peaks in the spectrum are all off scale. Clear structure is observed up to at least $1000 \mathrm{~cm}^{-1}$ above the $S_{1}$ origin.

tially ions that have one quantum of the corresponding excited vibration are formed. The frequency of this vibration in the ion is just a few $\mathrm{cm}^{-1}$ larger than $280 \mathrm{~cm}^{-1}$.

The apparent IP of TPA, as deduced from the spectra shown in fig. 3 and from the absolute frequency of the origin of the $S_{1} \leftarrow S_{0}$ transition $\left(29521 \mathrm{~cm}^{-1}\right)$, is at $6.880 \mathrm{eV}$, in good agreement with a previously estimated value [17]. It should be noted that the measurements of the IP are performed in an electric field of approximately $300 \mathrm{~V} / \mathrm{cm}$, and the actual fieldfree value for the IP will be several thousandths of an $\mathrm{eV}$ higher [18].

From a measurement of the double resonance TPAion signal as a function of the time delay between the two pulsed lasers an upper limit of $6 \mathrm{~ns}$ is deduced for the lifetime of the TPA $S_{1}$ state; an exact value for the lifetime can not be determined in this way since the pulse length of each of the dye lasers is already 45 ns.

\subsection{Spectra of TPA-Ar, Kr van der Waals complexes}

We have reported previously that under slightly different desorption conditions, complexes between the laser desorbed molecules and the carrier gas can be produced, internally cooled and mass selectively detected [19]. $(1+1)$-REMPI spectra of TPA-Ar and TPA-Kr obtained that way are shown in figs. $1 \mathrm{~b}$ and $1 \mathrm{c}$, respectively. Both spectra are measured at the parent mass, i.e. at $285 \mathrm{amu}$ for TPA-Ar and at 329 amu for TPA-Kr, ${ }^{84} \mathrm{Kr}$ being the most abundant $\mathrm{Kr}$ isotope. In these spectra the intensity of the strongest peaks is about $200-500$ times smaller than of that in the TPA spectrum. The most striking observation in both the spectrum of TPA-Ar and of TPA-Kr is both the size and the sign of the spectral shift with respect to the TPA origin. Never before has a blue-shift been observed in the complex of an aromatic molecule with either one Ar or one $\mathrm{Kr}$ atom, let alone a blue-shift of over $200 \mathrm{~cm}^{-1}$.

One has to be careful in assigning the correct spectral features to the specific size cluster, even if mass selective detection is applied; higher TPA-Ar ${ }_{n}$ clusters will fragment and give rise to a signal on the TPA$\operatorname{Ar}_{m}$ mass $(m \leqslant n)$ as well. There are two approaches commonly used to overcome this problem. In the first approach care is taken not to detect the fragments of larger clusters, although they are still formed $[20,21]$. In the other approach, soft ionization (i.e. bringing the molecule just barely above the ionization limit) is applied to strongly limit the fragmentation process itself [ 14]. For this latter approach two different lasers would have to be used for TPA, as one-color $(1+1)$-REMPI of TPA can bring the ion about 3500 $\mathrm{cm}^{-1}$ above the IP. In our measurements of the cluster spectra we recorded the spectra of TPA- $\mathrm{Ar}_{n}, \mathrm{Kr}_{n}$ for $n=0-4$ simultaneously, thereby being able to trace the origin of the various lines. All the spectral features observed in figs. $1 \mathrm{~b}$ and $1 \mathrm{c}$ are due to the TPA complexed with one $\mathrm{Ar}, \mathrm{Kr}$, respectively. Although there was severe fragmentation of these complexes 


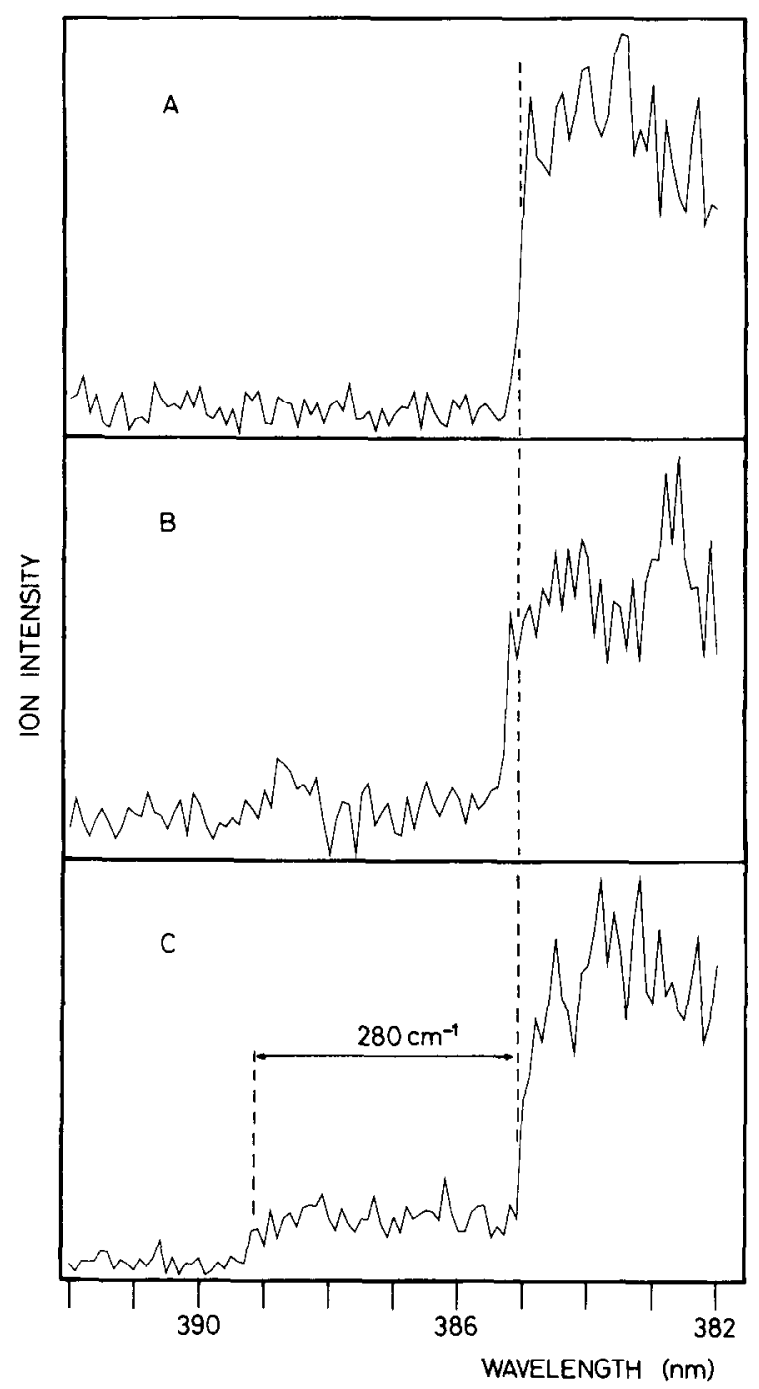

Fig. 3. Wavelength dependence of the TPA ion signal showing the onset of ionization after resonant excitation of TPA to (a) the vibrationless level in the $S_{1}$ state; (b) the $114 \mathrm{~cm}^{-1}$ vibration in the $S_{1}$ state; and (c) the $280 \mathrm{~cm}^{-1}$ vibration in the $S_{1}$ state.

(which made the spectra of these complexes appear just as strong at the TPA mass as at the parent mass of the complex) distinctly different spectral features were observed at the higher cluster masses.

About $30 \mathrm{~cm}^{-1}$ red-shifted from the TPA origin, the TPA-Ar spectrum, displayed in fig. $1 \mathrm{~b}$, shows a broad structure. As this is the position where we expected the origin of the $S_{1} \leftarrow S_{0}$ transition in the TPAAr complex to appear, i.e. it is the "normal" red- shifted position for an aromatic molecule complexed to an Ar atom, we tried very hard to optimize on this signal. There was no way, however, to get clearly resolved single sharp lines. Sharp structure does appear $211 \mathrm{~cm}^{-1}$ blue-shifted from the TPA origin. We believe this to be the origin of one of the possible, and apparently one of the most stable, TPA-Ar isomers. The TPA fundamental modes at 114 and $280 \mathrm{~cm}^{-1}$ are seen in the spectrum of the Ar complex as well, and from this a lower limit of $280 \mathrm{~cm}^{-1}$ can be deduced for the binding energy of this TPA-Ar isomer in the $S_{1}$ state.

There is another strong peak at $239 \mathrm{~cm}^{-1}$ to the blue of the TPA origin, which might be the origin of another isomer of TPA-Ar. Again, the main vibrational modes in the TPA molecule are built on top of this. Onc might also argue that this extra peak is due to a vdW Ar-TPA stretching mode at $28 \mathrm{~cm}^{-1}$. In that case, however, more of these modes should appear with comparable intensity, but they do not. In addition, the relative intensity of the 211 and 239 $\mathrm{cm}^{-1}$ shifted peaks changes with changing laser-desorption/jet-cooling conditions, i.e. with changing production conditions for the van der Waals complexes. Some extra peaks that we do observe at a slightly large frequency are attributed to $\mathrm{vdW}$ stretching modes of the TPA-Ar complex.

One might argue that the structure observed for the TPA-Ar complex to the blue of the TPA origin is due to a complex that is actually $69 \mathrm{~cm}^{-1}$ red shifted, and of which we only see the TPA vibrational progression $(-69+280=211)$. Although there is absolutely no signal visible at the thus predicted origin, we can also find strong experimental evidence against this conjecture by looking at the TPA- $\mathrm{Kr}$ complex. $\mathrm{Kr}$ has a larger polarizability than Ar (by a factor 1.5) and consequently a larger red-shift of the hypothetical origin (and of the TPA modes built on top of this) should be visible. Instead, a somewhat larger blueshift $\left(216 \mathrm{~cm}^{-1}\right)$ is observed for TPA-Kr than for TPA-Ar, ruling out this hypothesis. All in all the spectrum of TPA-Kr looks very similar to the TPAAr spectrum. There is some sign of a red-shifted isomer (somewhat further red-shifted than the TPA-Ar complex, as expected), but the strongest signal is from a $216 \mathrm{~cm}^{-1}$ blue-shifted isomer. Again, the two strongest TPA modes are seen in the $\mathrm{Kr}$ complex as well. There is a set of two peaks visible that are ap- 
proximately 21.5 and $26 \mathrm{~cm}^{-1}$ blue-shifted from the origin of this TPA-Kr complex. It might well be that one of them is actually the origin of another TPA-Kr isomer, while the other is due to a vdW TPA-Kr stretching mode.

We also measured the spectrum of the TPA-Ar complex, and this spectrum is shown in fig. 4, albeit on a different wavelength scale. The origin of the strongest TPA-Ar ${ }_{2}$ isomer was found at $29708 \mathrm{~cm}^{-1}$, about $24 \mathrm{~cm}^{-1}$ red-shifted with respect to the strong est TPA-Ar origin, but still with an overall blue-shift of $187 \mathrm{~cm}^{-1}$ with respect to the TPA origin. As indicated in the figure, the fundamental vibrations of TPA are seen in the spectrum of the $\mathrm{Ar}_{2}$ complex as well. Again, there are some extra peaks at low frequencies, 22 and $28 \mathrm{~cm}^{-1}$ further to the blue of the aforementioned TPA- $\mathrm{Ar}_{2}$ origin, which might either be due to vdW vibrations in the complex or due to other TPA$\mathrm{Ar}_{2}$ isomers.

A first conclusion from these measurements is that when Ar or $\mathrm{Kr}$ forms a complex with TPA, the first Ar, $\mathrm{Kr}$, atom goes preferentially in a "special" position, a position which causes an abnormal blue-shift of the spectrum. With the first rare-gas atom sitting in this special position, the second rare-gas atom is forced into a "normal" position, i.e. above one of the phenyl rings, causing a normal red-shift with respect to the TPA-Ar complex.

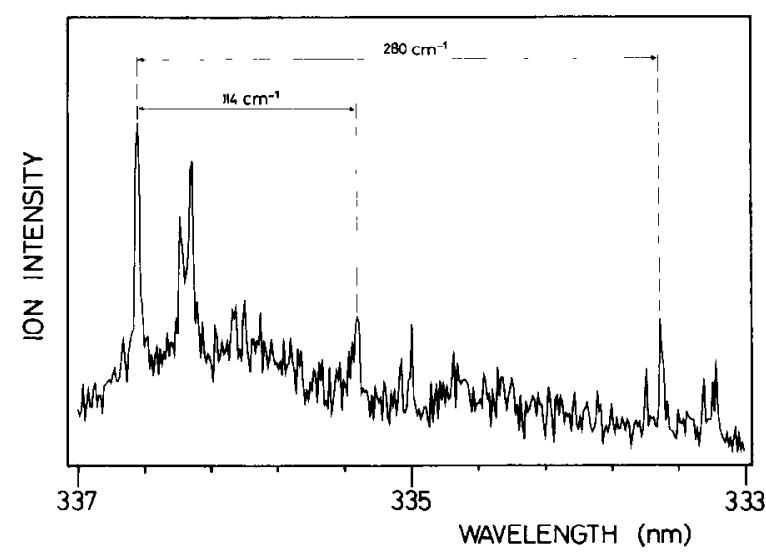

Fig. 4. $(1+1)$-REMPI spectrum of the TPA-Ar 2 complex. The indicated origin is $187 \mathrm{~cm}^{-1}$ blue-shifted from the origin of free TPA

\section{High-resolution LIF spectroscopy}

\subsection{Experimental setup}

In the Nijmegen laboratory high-resolution spectroscopy was performed to determine both the geometrical structure of TPA itself as well as that of the TPA-Ar complexes. Again, details of both the molecular beam machine as well as of the narrow bandwidth UV radiation source have been given elsewhere [22], and only a short description is given here.

TPA is heated in a quartz oven to approximately $200^{\circ} \mathrm{C}$ ( 10 Torr vapour pressure) and is expanded supersonically together with $0.6-1.0 \mathrm{~atm}$ Ar through a $0.15 \mathrm{~mm}$ nozzle, kept at a slightly higher temperature. The molecular beam is skimmed twice and enters a differentially pumped LIF detection chamber about $30 \mathrm{~cm}$ away from the beam orifice. There the molecular beam is crossed perpendicularly with the weakly focused UV laser beam. The TPA molecules are resonantly excited from the $S_{0}$ to the $S_{1}$ state and the total fluorescence back to the ground state is detected. The UV radiation is obtained by frequency doubling in a $\mathrm{LiIO}_{3}$ crystal inside the cavity of a single-frequency ring dye laser, operating on DCM. By pumping the dye laser with $6 \mathrm{~W}$ of an Ar ion laser (all lines), $0.2-0.5 \mathrm{~mW}$ of tunable radiation in the 339 $336 \mathrm{~nm}$ range with an effective bandwidth of about $3.0 \mathrm{MHz}$ is obtained. A single mode laser scan can be made over $50 \mathrm{GHz}$, enough to record the whole envelope of a single rovibronic band in the $S_{1} \leftarrow S_{0}$ electronic transition of TPA in a single scan. The TPA spectra are recorded together with the transmission peaks of a pressure and temperature stabilized interferometer with a free spectral range (in the UV) of $148.25 \pm 0.05 \mathrm{MHz}$. The absolute frequency of the transmission peaks of the interferometer, and thereby the absolute frequency of the TPA lines, is determined by the simultaneous recording of the $\mathrm{I}_{2}$ absorption spectrum in a cell at the fundamental laser frequency.Although the $I_{2}$ absorption spectrum is relatively sparse in this region, an accuracy better than $0.1 \mathrm{~cm}^{-1}$ can still be obtained for the absolute frequency, the error being mainly due to the determination of the centre of the broad and not always symmetric $\mathrm{I}_{2}$-lines. Relative line positions are measured to an accuracy of $10 \mathrm{MHz}$. 


\subsection{The rotationally resolved spectrum of $T P A$}

In the top portion of fig. 5 the measured high-resolution spectrum of the origin of the TPA $S_{1} \leftarrow S_{0}$ transition is shown. The absolute frequency of the origin ( 0.0 on the scale in the figure) is at $\nu_{\text {vac }}=29520.7$ $\mathrm{cm}^{-1}$. The signal intensity of the strongest peak is around 5000 counts/s, whereas there is a continuous background of about 200 counts/s due to scattered laser radiation. This high-resolution spectrum is clearly that of a parallel transition of a symmetric top molecule. There is a strong Q-branch, blue degraded, an R-branch and a P-branch with a band head. The lower portion of fig. 5 shows a fit to the data. The formulas for a rigid oblate symmetric top molecule are used and these fit the data perfectly well. The constants we used are the rotational constant $B^{\prime \prime}$ in the $\mathrm{S}_{0}$ state and $\Delta B$ and $\Delta C$, the differences of constants

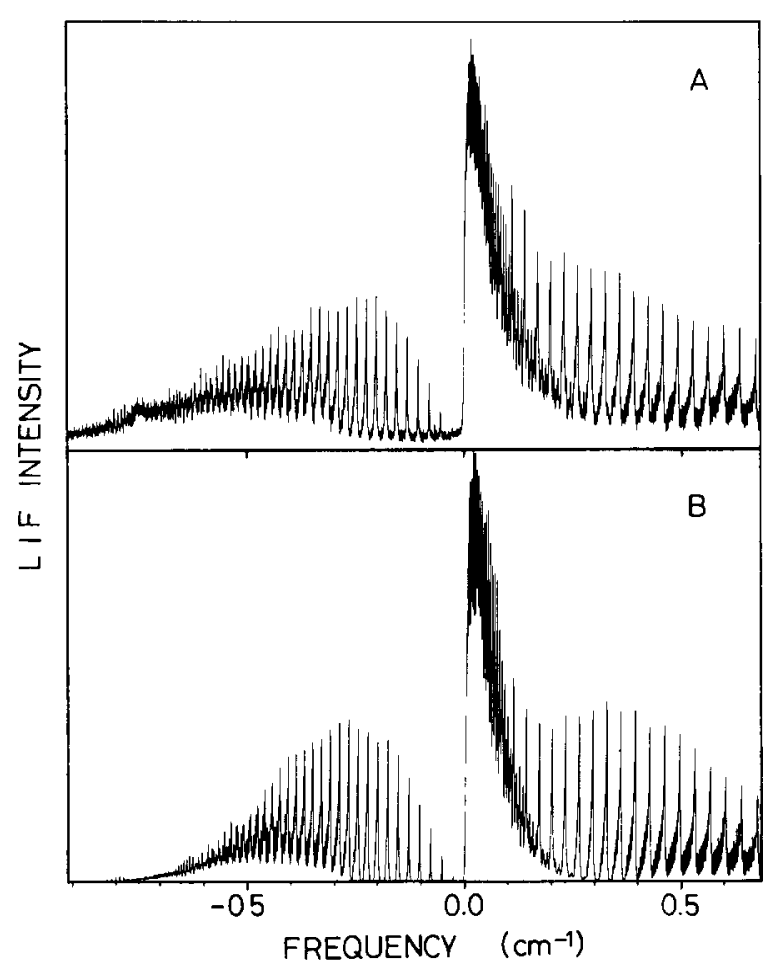

Fig. 5. High-resolution LIF spectrum of the origin of the $S_{1} \leftarrow S_{0}$ transition in jet-cooled TPA. The experimentally observed spectrum (a) can be fitted into the smallest detail, using the formulas for a rigid oblatc symmetric top molcculc, as shown in (b). Notc the band head in the P-branch for large $J^{\prime \prime}, K^{\prime \prime}$ values. in the $S_{1}$ and $S_{0}$ state. The rotational constant determined by the moment of inertia around the symmetry axis of TPA, the $c$-axis, cannot be determined in a parallel transition of a symmetric top molecule. The values that we used in the fit are given in table 1 . It should be noted that the values of $\Delta B$ and $\Delta C$ are much more precise than the value of $B^{\prime \prime}$; the inaccuracy in the latter constant is due to a slight drift of the interferometer during the scan of the spectrum and is the standard deviation in the value of $B^{\prime \prime}$ obtained on various days, scanning in either frequency direction. For the $C^{\prime \prime}$ constant a value of $230 \mathrm{MHz}$ was assumed, based on an estimate of the geometrical structure of TPA, as will be discussed later. The exact value for this constant is not critical anyway; it only influences to a minor extent the intensities of the various lines. A perpendicular transition, which is intrinsically weak in the TPA $S_{1} \leftarrow S_{0}$ band, has to be measured to determine the absolute value of $C^{\prime \prime}$ in TPA. To obtain the fit shown in fig. $5 b$, a rotational temperature of $4.0 \mathrm{~K}$ was assumed, a value that we typically find for the rotational temperature in large aromatic molecules using this molecular beam apparatus. $J$-values up to $J=100$ were included in the fit; to reproduce the observed band head in the P-branch more accurately even higher $J$-values have to be included.

In fig. 6 an enlargement of a part of the experimental (upper) and fitted (lower) R-branch of TPA is shown. Individual $J, K$ lines are resolved. In the fit a Lorentzian lineshape with a full width at half maximum (fwhm) of $40 \mathrm{MHz}$ is assumed. The contribution to the linewidth due to residual Doppler broadening in the molecular beam (i.e. the Gaussian

Table 1

Rotational constants for TPA and TPA-Ar as determined from the high-resolution LIF spectrum of the origin of the TPA $S_{1} \leftarrow S_{0}$ transition at $29520.7 \mathrm{~cm}^{-1}$ and of the $211 \mathrm{~cm}^{-1}$ blue-shifted origin of TPA-Ar at $29731.2 \mathrm{~cm}^{-1}$

\begin{tabular}{lcc}
\hline $\begin{array}{l}\text { Rotational constants } \\
(\mathrm{MHz})\end{array}$ & TPA & TPA-Ar \\
\hline$B^{\prime \prime}$ & $403.7 \pm 0.5$ & $280 \pm 5$ \\
$C^{\prime \prime}$ & $230^{\mathrm{a})}$ & $230^{\mathrm{b})}$ \\
$\Delta B \equiv B^{\prime}-B^{\prime \prime}$ & $7.4 \pm 0.1$ & $-8.5 \pm 0.5$ \\
$\Delta C \equiv C^{\prime}-C^{\prime \prime}$ & $2.8 \pm 0.1$ & $2.8^{\mathrm{b}}$ \\
\hline
\end{tabular}

a) Calculated value for a TPA geometry with $\phi=50.0^{\circ}$ and $\angle \mathrm{CNC}=120.0^{\circ}$.

b) Kept fixed to the value as obtained for TPA. 


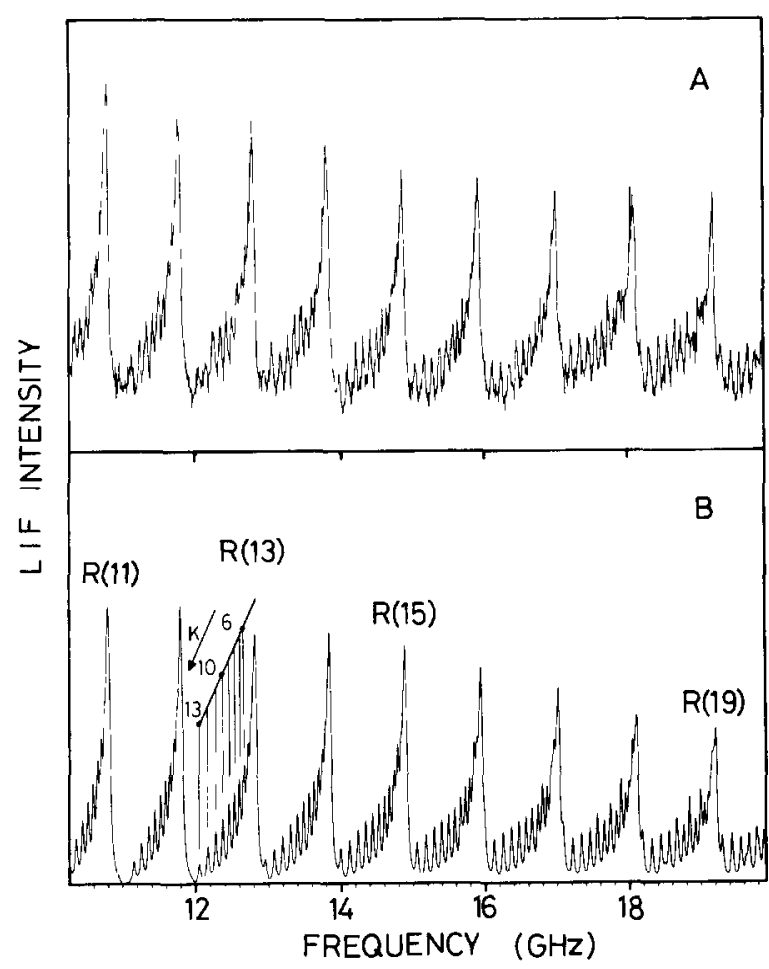

F1g. 6. Part of the R-branch of the experimental (a) and fitted (b) spectrum of TPA. As explicitly indicated in the figure, ind1vidual $J, K$ lines can be resolved and assigned. The width (fwhm) of individual lines is about $40 \mathrm{MHz}$.

contribution to the linewidth) is known to be 12 MHz. The largest contribution to the observed linewidth therefore has to come from lifetime broadening. Deconvolution of the experimentally observed Voigt profile yields a Lorentzian contribution to the linewidth of around $36 \mathrm{MHz}$, implying a lifetime for individual $J^{\prime}, K^{\prime}$ levels in the vibrationless $\mathrm{S}_{1}$ state of TPA of $4.5 \pm 0.5 \mathrm{~ns}$.

In the fit to the experimental data the spin statistical weights have not been taken into account, as they are very close to one with five fermions present in each phenyl ring. No splitting is observed due to the isomeric right-hand rotating and left-hand rotating forms of TPA.

In addition to the origin of the $S_{1} \leftarrow S_{0}$ transition we also measured the transitions to the 114 and $280 \mathrm{~cm}^{-1}$ vibrations under high resolution. We found the same rotational constants within the quoted error bars. As the spectrum of the transition to either one of these vibrations is also that of a parallel transition of TPA, both vibrations are due to symmetric modes.

\subsection{High-resolution spectra of TPA-Ar}

To determine the geometrical structure of especially the blue-shifted TPA-Ar complex we measured its spectrum under high resolution, as had been done previously for other vdW clusters between aromatic molecules and rare-gas atoms in the same molecular beam apparatus $[23,24]$. The best experimental spectrum which we managed to obtain reproducibly for the $211 \mathrm{~cm}^{-1}$ blue-shifted TPA-Ar isomer is shown in the upper part of fig. 7. Although it looks hopeless at first sight, there is some clear structure visible that we managed to simulate, as shown in the lower portion of the figure. Most importantly, the observed TPA-Ar spectrum can be simulated using the formulas for a rigid symmetric top.

In our simulation we assume the Ar atom to be located on the $c$-axis of the TPA molecule. Then neither $C^{\prime \prime}$ nor $\triangle C$ will change in going from TPA to TPA-Ar. Furthermore, we assume the individual lines to have a Lorentzian shape with a width (fwhm) of $40 \mathrm{MHz}$, just as for the free TPA. A rotational temperature of $5.0 \mathrm{~K}$ is taken for the simulation of the

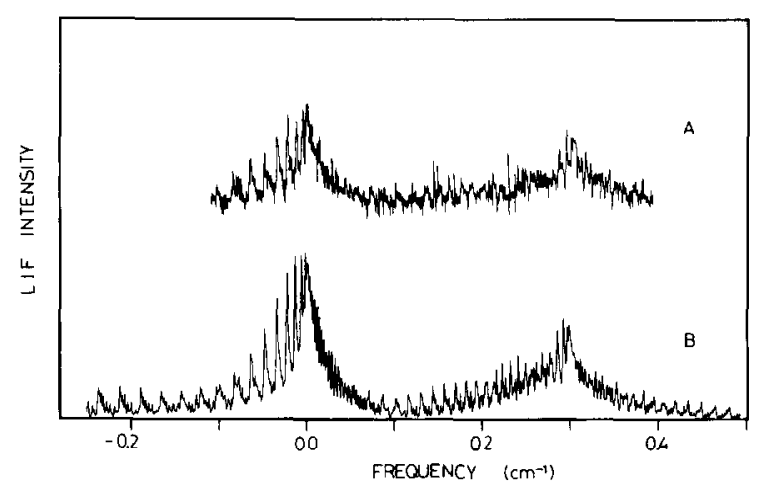

Fig. 7. Experimentally observed (a) and sımulated (b) high-resolution LIF spectrum of the origin of the $211 \mathrm{~cm}^{-1}$ blue-shifted TPA-Ar complex at $29731.2 \mathrm{~cm}^{-1}$. The simulated TPA-Ar spectrum is for a van der Waals complex that is a symmetric top, i.e. a vdW complex in which the Ar atom is sitting on the $c$-axis. In the simulation the same rotational temperature $(4.0 \mathrm{~K})$, the same lineshape (Lorentiian, $40 \mathrm{MHz} \mathrm{fwhm}$ ) and the same values for $C^{\prime \prime}$ and $\triangle C$ were taken as found for TPA. The simulated spectrum is for $B^{\prime \prime}=280 \pm 5 \mathrm{MHz}$ and $\Delta B=-8.5 \pm 0.5 \mathrm{MHz}$. As now $\Delta B$ is negative, the $\mathrm{R}$-branch has a band head and the Q-branch degrades to the red. 
TPA-Ar complex. This leaves only two constants that have to be varied, $B^{\prime \prime}$ and $\Delta B$, of which $B^{\prime \prime}$ can be reasonably estimated from the calculated position of the $\mathrm{Ar}$ atom on the $c$-axis in the minimum energy configuration, as will be discussed in section 4.3. Because the observed blue-shift indicates that the TPAAr complex is less bound in the excited state than in the ground state, one expects the $\mathrm{N}-\mathrm{Ar}$ distance to increase upon $S_{1} \leftarrow S_{0}$ excitation, producing a negative value of $\Delta B$.

Having only these two parameters to vary, the simulated spectrum shown in fig. $7 \mathrm{~b}$ is obtained with $B^{\prime \prime}=280 \mathrm{MHz}$ and $\Delta B=-8.5 \mathrm{MHz}$, as also indicated in table 1 . Since $\Delta B$ is negative, the R-branch shows a band head, whereas the Q-branch runs into the P-branch. The apparently single sharp lines around the origin are due to a coincidental bunching of a large number of lines, and no individual $J^{\prime \prime}, K^{\prime \prime}$ assignments can be made. The absolute frequency of the origin of the spectrum of this TPA-Ar isomer is $29731.2 \mathrm{~cm}^{-1}$, so the spectrum of this isomer is blueshifted by $210.5 \mathrm{~cm}^{-1}$ with respect to the transition in TPA. The total number of lines included in the simulated part of the spectrum that is shown is about 5000 .

Up to now we did not succeed in measuring the high-resolution spectrum of the (possibly) TPA-Ar isomer that is even $28 \mathrm{~cm}^{-1}$ further blue-shifted nor of the less-structured red-shifted TPA-Ar isomer. Both in view of the low signal to noise ratio on the TPA-Ar complex that we did observe, as well as in view of the fact that the production conditions in the cw beam machine are very different from those in the laser-desorption jet-cooling apparatus, this is not too surprising.

\section{Discussion}

\subsection{The geometrical structure of TPA}

As already mentioned in section 1 there is some ambiguity in the literature on the geometrical structure of free TPA in the gas phase. The high-resolution spectra reported here give a straight answer to this question: within our present accuracy of $0.1 \mathrm{MHz}$, two of the rotational constants for TPA are identical, implying a symmetric top structure for TPA. This means that the TPA molecule has at least $\mathrm{C}_{3}$ symmetry. For the rest of the discussion we will therefore use the geometry of TPA as depicted in fig. 8 .

The three phenyl rings are assumed to be flat and identical to each other, with fixed C-C $(1.379 \AA)$ and $\mathrm{C}-\mathrm{H}(1.070 \AA)$ distances and $\angle \mathrm{CCC}$ angles and $\angle \mathrm{CCH}$ angles of exactly $120^{\circ}$. The values for the bondlengths are the averaged values as obtained from an X-ray crystallographic study of TPA in the solid [6]. Then there are only three other parameters left that are allowed to vary, i.e. the $\mathrm{C}-\mathrm{N}$ distance (taken as $1.419 \AA$ in the $\mathrm{S}_{0}$ state of TPA), the angle $\phi$ by which the phenyl groups are rotated around the $\mathrm{C}-\mathrm{N}$ bond and the $\angle \mathrm{CNC}$ angle. The angles are defined such that a fully planar geometry of TPA means $\phi=0$ and $\angle \mathrm{CNC}=120^{\circ}$. If $\angle \mathrm{CNC}$ is exactly $120^{\circ} \mathrm{TPA}$

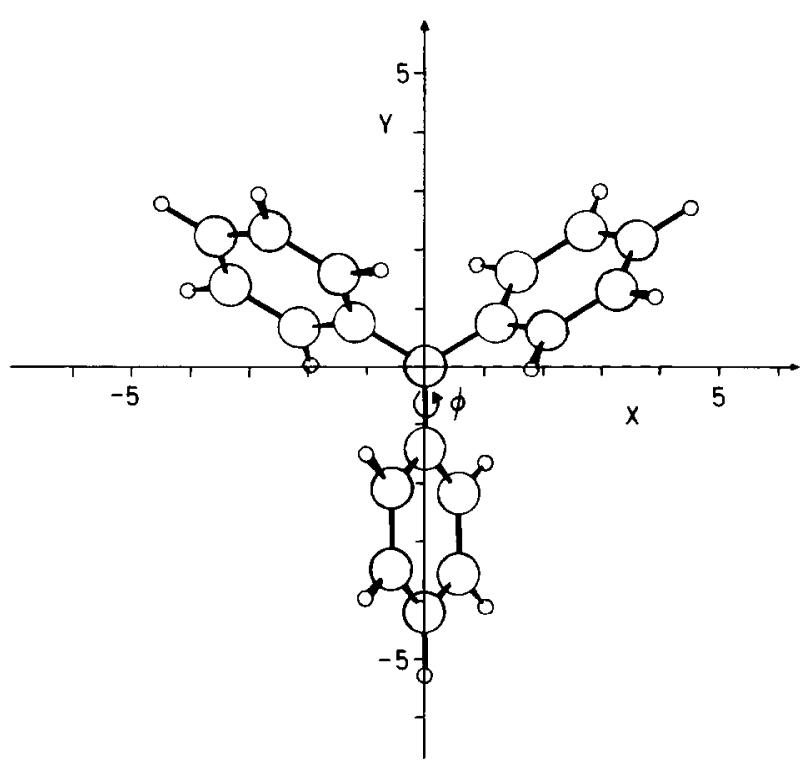

Fig. 8. Schematic of the TPA geometry and the axes system used in the calculation of the TPA-Ar potential energy surface. The $\mathrm{N}$ atom is taken to be in the origin of a right handed $(x, y, z)$ coordinate system. The scale along the $x$ - and $y$-axis is in $\AA$. All three phenyl rings are rotated in a symmetric way around the $\mathrm{C}-\mathrm{N}$ bonds by an angle $\phi$. The angle $\phi$ is defined such that $\phi=90^{\circ}$ means that all three phenyl rings are perpendicular to the plane of the paper. If $\angle \mathrm{CNC}=120^{\circ}$ the molecule looks the same from the top (positive z-values) and the bottom (negative z-values). For $\angle \mathrm{CNC}$ smaller than $120^{\circ}$, the phenyl rings are bent down, so that positive $z$-values correspond to a position above the umbrella whereas negative $z$-valucs correspond to positions underneath the umbrella. 
has additional $\mathrm{C}_{2}$ symmetry axes through each of the three phenyl rings, yielding a $D_{3}$ symmetry for the molecule. In the literature up to now no definite values for either $\phi$ or $\angle \mathrm{CNC}$ have been obtained, although averaging of the existing literature values would give a $\phi$ somewhere between $15^{\circ}$ and $60^{\circ}$ and a $\angle \mathrm{CNC}$ angle that is quite large, between $120^{\circ}$ and $114^{\circ}$.

The $S_{1} \leftarrow S_{0}$ transition in TPA is probably best described as a $\pi^{*} \leftarrow n$ transition, which means that if TPA is electronically excited from $S_{0}$ to $S_{1}$, one of the lone pair electrons on the $\mathrm{N}$ atom is moved towards the phenyl rings. Therefore, upon excitation a change is mainly expected in the bonds and the angles involving the $\mathrm{N}$ atom. More specifically, the $\mathrm{C}-\mathrm{N}$ bonds are expected to shorten and to get stiffer upon $S_{1} \leftarrow S_{0}$ excitation.

The vibrationally resolved electronic $S_{1} \leftarrow S_{0}$ spectrum of TPA, shown in fig. la, shows a progression in two low-frequency modes, indicating a substantial change in the geometry of TPA upon excitation. The $280 \mathrm{~cm}^{-1}$ mode, which we know to be symmetric from its high-resolution spectrum, is most likely due to the symmetric $\mathrm{N}$-phenyl stretch mode. This modc has been observed at $290 \mathrm{~cm}^{-1}$ in Raman spectra of TPA in a $\mathrm{CS}_{2}$ solution [2]. More recently, the frequency of this mode has been calculated in the $S_{0}$ state as well as in the ground state of the positive ion of TPA to be around $300 \mathrm{~cm}^{-1}$ [25]. A long progression in this mode is in full agreement with the expected shortening of the $\mathrm{C}-\mathrm{N}$ bonds upon excitation. The other low-frequency vibrational mode at 114 $\mathrm{cm}^{-1}$ is also symmetric and is most likely due to the symmetric torsion of the phenyl rings around the C$\mathrm{N}$ bond. The frequency of this mode has also been calculated [25], and there is a large difference between the calculated frequency of this mode in the $S_{0}$ state of the neutral $\left(74 \mathrm{~cm}^{-1}\right)$ and in the ground state of the positive ion $\left(104 \mathrm{~cm}^{-1}\right)$. From the IP measurements as described in section 2.2 we know that the frequency of the mode corresponding to the 114 $\mathrm{cm}^{-1}$ vibration in the $S_{1}$ state is around $104 \mathrm{~cm}^{-1}$ in the ground state of the ion. Apparently the frequency for the symmetric torsion mode increases substantially in going from $S_{0}$ to $S_{1}$, indicating an increased stiffness in the $\mathrm{C}-\mathrm{N}$ bonds in the excited state. The observed Franck-Condon intensity pattern in the progression of this mode is in agreement with a change in the angle $\phi$ of $2^{\circ}$ to $3^{\circ}$ in going from $S_{0}$ to $S_{1}$. A Franck-Condon analysis does not give the sign of this change.

In the recent theoretical calculation [25] two lowfrequency modes are found for TPA in the $\mathrm{S}_{0}$ state around $20-25 \mathrm{~cm}^{-1}$. In the ground state of the ion these modes are at a much larger frequency, around $45-50 \mathrm{~cm}^{-1}$. It is expected that the frequency of the corresponding modes in the $S_{1}$ state of TPA is close to the frequency of those in the ion. Under our experimental conditions these low-frequency modes are certainly populated to some extent and transitions from these nonsymmetric low-frequency modes in the $\mathrm{S}_{0}$ state to the corresponding modes in the $\mathrm{S}_{1}$ state will be blue-shifted with respect to the TPA origin. The 23 and $32 \mathrm{~cm}^{-1}$ blue-shifted lines that are observed in the TPA spectra are therefore probably hotbands, as mentioned in section 2.2.

The rotational constants for TPA, as determined from the high-resolution spectroscopy of TPA and as given in table 1 , can be fitted to several geometries. If it is assumed that only the $\mathrm{C}-\mathrm{N}$ bondlength and the angle $\phi$ change upon excitation, then $\Delta B$ and $\Delta C$ indicatc a decrcase of the angle $\phi$ of $2^{\circ}$ to $3^{\circ}$ and a decrease in the $\mathrm{C}-\mathrm{N}$ bond length of $0.03 \AA$ in going from $S_{0}$ to $S_{1}$. This change in $\phi$ is in perfect agreement with the observed Franck-Condon intensity distribution in the progression for the symmetric torsion mode. The change in $\mathrm{C}-\mathrm{N}$ bond length is very moderate, compared with for instance the situation in aniline [14]. The absolute value of $B^{\prime \prime}$ can be fitted to a set of $(\phi, \angle C N C)$ angles, as indicated in table 2. For all sets the changes in $\phi$ and the $\mathrm{C}-\mathrm{N}$ bond length are as mentioned before. As seen in table 2 , if the $\mathrm{N}$ atom is in the plane of the three adjacent $\mathrm{C}$ atoms the angle $\phi$ is $50.0^{\circ}$. A more pyramidal structure of TPA is only in agreement with the measured $B^{\prime \prime}$ value if at the same time the angle $\phi$ increases, i.e. if at the same time the phenyl rings turn towards a more staggered geometry. Instead of the $\angle \mathrm{CNC}$ often the "umbrellaangle" $\Theta$, i.e. the angle by which the $\mathrm{C}-\mathrm{N}$ bonds are bent out of the $x y$-plane, is used. These angles are related via: $\cos (\angle \mathrm{CNC})=1-\frac{3}{2} \cos ^{2} \theta$.

\subsection{The geometry of the various $T P A-A r, K r$ isomers}

For the $210.5 \mathrm{~cm}^{-1}$ blue-shifted TPA-Ar isomer the $\mathrm{Ar}$ is located on the $\mathrm{C}_{3}$ axis, as is unambiguously 
Table 2

In the first column, various possible $(\phi, \angle C N C)$ combinations that yield a rotational constant along the $b$-axis that is in agreement with the experimentally found value for $B^{\prime \prime}$ are given. In the electronic ground state of TPA, $\phi$ and $\angle$ CNC are the only variables; for all the bond lengths as well as for all the other angles, values as obtained from X-ray analysis of solid TPA are taken. In the second and third column the calculated minimum value for the TPA-Ar van der Waals potential on the $z$-axis as well as the distance of the Ar atom to the $\mathrm{N}$ nucleus are given for an Ar atom above and underneath the umbrella, respectively. In the last column the measured value for the Ar position, i.e. the position as deduced from the measured $B^{\prime \prime}$ value for the complex, is indicated

\begin{tabular}{|c|c|c|c|}
\hline $\begin{array}{l}\text { Possible }(\phi, \angle C N C) \\
\text { combinations } \\
\text { (deg.) }\end{array}$ & $\begin{array}{l}\text { TPA-Ar vdW min. } \\
\text { above umbrella } \\
\left(\mathrm{cm}^{-1}, \AA\right)\end{array}$ & $\begin{array}{l}\text { TPA-Ar vdW min } \\
\text { under umbrella } \\
\left(\mathrm{cm}^{-1}, \AA\right)\end{array}$ & $\begin{array}{l}\text { Ar position } \\
( \pm 0.10 \AA) \\
(\AA, \AA)\end{array}$ \\
\hline$(50.0,120.0)$ & $-438,+4.22$ & $-438,-4.22$ & $+4.01,-4.01$ \\
\hline$(54.5,119.0)$ & $-391,+4.24$ & $-420,-4.56$ & $+3.74,-4.28$ \\
\hline$(59.0,118.0)$ & $-359,+4.34$ & $-400,-4.78$ & $+3.63,-4.39$ \\
\hline$(64.0,117.0)$ & $-331,+4.44$ & $-383,-4.98$ & $+3.54,-4.48$ \\
\hline$(70.0,116.0)$ & $-307,+4.56$ & $-367,-5.18$ & $+3.47 .-4.55$ \\
\hline$(80.0,115.0)$ & $-284,+4.69$ & $-351,-5.38$ & $+3.41,-4.62$ \\
\hline
\end{tabular}

shown by the high-resolution spectrum of this complex. There are of course two positions possible, one underneath and one above the umbrella; these positions are equivalent if $\angle \mathrm{CNC}$ equals $120^{\circ}$. The positions for the Ar atom on the $\mathrm{C}_{3}$ axis as deduced from the measured $B^{\prime \prime}$ value for the TPA-Ar complex are indicated in the last column of table 2 . The exact positions are of course strongly dependent on the choices that are made for the angles $(\phi, \angle \mathrm{CNC})$. In all cases, however, the magnitude and sign of $\triangle B$ for TPA-Ar imply that in the electronically excited state of the complex the Ar atom has moved considerably further away from the $\mathrm{N}$ atom, approximately by $0.20 \pm 0.02$ $\AA$.

Qualitatively, the fact that the Ar atom moves further away from the $\mathrm{N}$ atom upon electronic excitation is in agreement with the observed blue-shift of the spectrum; both facts indicate that the Ar atom is less bound in the $S_{1}$ state than in the $S_{0}$ state.

The question remains, however, which effect causes this abnormal blue-shift. As the electronic transition in TPA is probably best described as a $\pi^{*} \leftarrow n$ transition, there is a larger electron density on the $C_{3}$ axis, due to the lone pair electrons of the $\mathrm{N}$ nucleus, in the ground state of the TPA molecule than in the electronically excited state. Therefore a stronger overlap of the Ar atom with the electron density of TPA is expected in the ground state than in the electronically excited state. If attractive forces between the Ar atom and the TPA molecule are more important than the repulsive contributions, this would explain the sign of the observed electronic shift of the TPA-Ar complex. An explanation analogous to the one given by Hermine et al. to explain the blue-shift for one of the observed aniline-Ar ${ }_{3}$ isomers [14] holds for the TPA-Ar complex; the Ar atom sitting on the $\mathrm{C}_{3}$ axis slightly hinders the electron density on the $\mathrm{N}$ nucleus to be hybridized with the $\pi$ system of the phenyl rings as much as it would like to, i.e. as much as it is in the free TPA. This then causes a kind of a chemical shift in the complexed TPA, bringing its spectrum more in the direction of the lowest singlet-singlet transition in benzene, this is, to the blue. It should be noted that the shift of TPA-Ar relative to TPA is about $2.5 \%$ of the shift of TPA relative to benzene.

\subsection{Calculations on the minimum energy TPA-Ar complex geometries}

To rationalize the position of the Ar atom on the $\mathrm{C}_{3}$ axis in TPA, as well as to rationalize the apparent existence of various TPA-Ar isomers, the potential energy surface for an Ar atom around a TPA molecule in its electronic ground state is calculated. For this, atom-atom potential of the Lennard-Jones type are summed over all the atoms in TPA, as prescribed by Ondrechen et al. [26]. For the ground state of TPA interacting with an Ar atom, we use the same C-Ar, $\mathrm{H}-\mathrm{Ar}$ and $\mathrm{N}$-Ar interaction parameters as used in the calculation of aniline- $\mathrm{Ar}_{n}$ cluster geometries [13]. The geometry of TPA in the electronic ground state is taken as discussed in section 4.1 and as shown in 
fig. 8. For all the different $(\phi, \angle \mathrm{CNC})$ combinations shown in table 2, the two-dimensional minimum energy surface for an Ar atom attached to the TPA molecule is calculated. The plane defined by the $\mathrm{N}$ atom and the three adjacent $\mathrm{C}$ atoms (if $\angle \mathrm{CNC}$ is $120.0^{\circ}$ ) is taken to be the $x y$-plane. The $z$-axis is along the $\mathrm{C}_{3}$ symmetry axis of TPA, positive $z$-values indicating a position above the umbrella. In (more or less) planar molecules, a two-dimensional picture of the binding energy versus position of the Ar atom relative to the molecular framework is often given by taking a cut through the potential energy surface at a certain height above the molecule. In the case of TPA this would not make any sense, as the molecule is not planar at all. Therefore, the two-dimensional contour plots shown for two different sets of possible $(\phi, \angle \mathrm{CNC})$ angles in fig. 9 , are $2 \mathrm{D}$ projections of the $3 \mathrm{D}$ minimum energy surface on the $x y$-plane. The minimum energy values in a certain point $(x, y)$ are calculated along the $z$-coordinate, from $z=\infty$ to $z=0$ and from $z=0$ to $z=-\infty$. The values for the energy minima thus obtained are plotted in a contour plot in the left and right column of fig. 9 , respectively. Different values of $z$ belong to each of the points in the figure. Contour lines are drawn in $40 \mathrm{~cm}^{-1}$ intervals, and the contour lines at $-320 \mathrm{~cm}^{-1}$ are indicated in each of the plots.

A first inspection of fig. 9 shows that it is indeed energetically favourable for the Ar atom to sit on the $\mathrm{C}_{3}$ symmetry axis of the TPA molecule, although there
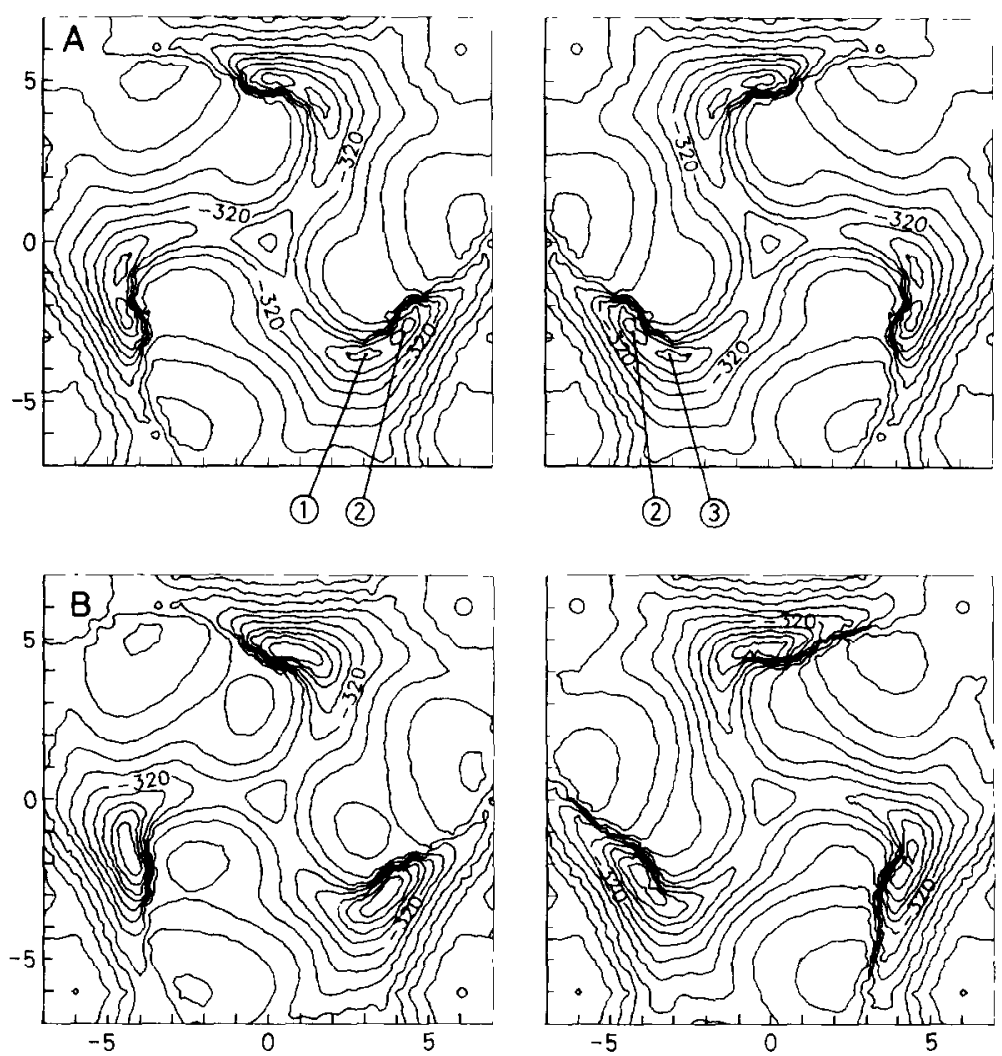

Fig. 9. Topographic plots of the TPA-Ar potential energy surface for two possible $(\phi, \angle \mathrm{CNC})$ combinations. For the combination $\mathrm{A}$ $\left(50.0^{\circ}, 120.0^{\circ}\right)$ and $B\left(59.0^{\circ}, 118.0^{\circ}\right)$ the minimum energy for the TPA-Ar complex for a certain value of $(x, y)$ is calculated as a function of $z$, for $z$-values from $+\infty \rightarrow 0$ (left column) and for $z$-values from $0 \rightarrow-\infty$. So the left column shows contour plots for positive $z$-values (positions above the umbrella) whereas in the right column the corresponding TPA-Ar potential energy surface for negative $z$ values is projected on a $2 \mathrm{D}$ plane. Contour lines are $40 \mathrm{~cm}^{-1}$ apart. 
are also some other, and even deeper, minimum energy configurations possible. In fig. 9a the minimum energy on the $C_{3}$ symmetry axis is $-438 \mathrm{~cm}^{-1}$ and this value is obtained for a $z$-value of $z= \pm 4.22 \AA$. The even deeper off-axis minima, indicated with number (1), (2) and (3), belong to $z$-values closer to the $x y$-plane. The minimum numbered $(1)$ is for a $z$-value around $z=+1.0 \AA$ and the minimum numbered ( 3 ) is an equivalent minimum on the other side of the TPA, so for $z=-1.0 \AA$; since in fig. $9 \mathrm{a} \angle \mathrm{CNC}$ is equal to $120^{\circ}$, positions above and below the TPA are identical. The equivalent minima numbered (2) are in the $z=0$ plane and these act as "gateways" for the Ar atom to move to the other side of the molecule. A closer inspection of the contour plots learns that the minimum energy surface is critically dependent on the chosen TP $\Lambda$ geometry. In going from the flat geometry (fig. 9a) towards a more umbrella like geometry (fig. 9b) several features change. First of all, as $\angle \mathrm{CNC}$ decreases, the "top" and "bottom" of TPA are no longer identical. The minimum on the symmetry axis for both positive and negative values of $z$ becomes less deep, distinctively more so for the minimum on top of the umbrella (positive $z$-valucs) than for the one underneath. (Note that the first contour line away from the $C_{3}$ axis in fig. $9 b$ is at -320 and at $-360 \mathrm{~cm}^{-1}$ for the left and right column, respectively.) Secondly, the various minima between the phenyl rings gradually merge together and get deeper and deeper, much deeper than the minima on the $\mathrm{C}_{3}$ axis. For $(\phi, \angle \mathrm{CNC})$ combinations further down in table 2 , first the minimum on the $C_{3}$ axis above the umbrella, but the eventually also the minimum on the $\mathrm{C}_{3}$ axis underneath the umbrella, completely disappears.

Care should be taken in the interpretation of these contour plots. The Lennard-Jones model is too simplistic to describe the complicated vdW interactions. The atom-atom interaction parameters have been used extensively and have been optimized for a number of aromatics, but in practically all of these systems the rare-gas atom is located more or less above the centre of a benzene ring. It has been pointed out recently [27] that in this case the simple LennardJones atom-atom potential model works remarkably well, but that this is no longer true when the rare-gas atom is further off to the side of the benzene plane. In the case of TPA-Ar, minima are found either on the symmetry axis, i.e. far away from the centrelines through the phenyl rings, or above one of the phenyl rings. For the latter minima the interaction with one of the rings is probably described quite correctly, but this is certainly not the case for the interaction with the other two rings. Therefore, care should be taken especially when the relative depths of the various minima for the TPA-Ar complex are compared, since now binding to different positions in the TPA molecule is compared.

The position of the various minima is more likely to be correct, however, and it is clear from the calculations that, generally speaking, only two types of TPA-Ar complexes will be possible, i.e. either with the Ar atom on the $\mathrm{C}_{3}$ symmetry axis or with the $\mathrm{Ar}$ atom above one of the phenyl rings. Experimentally we do observe the isomer with the $\mathrm{Ar}$ on the $\mathrm{C}_{3}$ axis to be the most abundant (this is the blue-shifted complex ), although there is a sign of the presence of the other type of TPA-Ar complex as well (the slightly red-shifted complex ).

For each set of $(\phi, \angle \mathrm{CNC})$ indicated in table 2 , the depth of the calculated potential energy minima on the $\mathrm{C}_{3}$ axis as well as the $z$-coordinate for which these minima appear are given in the second and third column. The measured Ar-N distance, i.e. the Ar-N distance inferred from the high-resolution LIF spectrum of the $211 \mathrm{~cm}^{-1}$ blue-shifted TPA-Ar isomer, is given in the last column of table 2 . Of course, two positions are always possible, and the fitted Ar-N distance is dependent on the chosen TPA geometry. Best agreement between the calculated and observed Ar position is obtained if a planar TPA geometry is assumed, although a slightly bent TPA geometry cannot be ruled out.

\section{Conclusions}

The rotationally resolved spectrum of the $S_{1} \leftarrow S_{0}$ transition of jet-cooled TPA is the spectrum of a symmetric top molecule. The identical phenyl rings are rotated around the $\mathrm{C}-\mathrm{N}$ bonds out of the planar geometry over an angle $\phi$ of about $50^{\circ}$. The $\angle \mathrm{CNC}$ angle is close to or equal to $120^{\circ}$ for gas phase TPA. Upon electronic $S_{1} \leftarrow S_{0}$ excitation, there is only a minor change in the geometry of the molecule; the $\mathrm{C}-\mathrm{N}$ bond length decreases by approximately $2 \%$ whereas 
the phenyl rings rotate over $2-3^{\circ}$ towards a flatter geometry.

The spectrum of the most abundant TPA-Ar complex shows an abnormal blue-shift with respect to the spectrum of the free TPA molecule. The high-resolution spectrum of this blue-shifted complex is again that of a symmetric top molecule, indicating that the Ar atom is located on the $\mathrm{C}_{3}$ symmetry axis of TPA. The spectrum of another possible TPA-Ar complex shows a "normal" red-shift, and in this case the Ar atom is most likely located above one of the phenyl rings.

The detailed interaction mechanism responsible for the observed blue-shift of the TPA-Ar complex is not understood yet.The TPA molecule, being a symmetric top molecule, should be perfectly suited to study the effect of an aromatic-molecule-rare-gas interaction in which the rare-gas atom is bound to a less common place of the aromatic molecule. Moreover, the data presented here can be used to test various (empirical) models for the vdW interactions.

\section{Acknowledgement}

GM gratefully acknowledges the support of the Dutch Royal Academy of Science (KNAW). This work was made possible in part by financial support from the Nederlandse Organisatie voor Wetenschappelijk Onderzoek (NWO).

\section{References}

[1] J. Sasak1, K. Kımura and M. Kubo. J. Chem. Phys. 31 (1959) 477.

[2] A.N. Rodionov, N.I. Ruch'eva, K.L. Rogozhın and D.N. Shigorın. Zh. Prikl. Spektrosk. 20 (1974) 534.

[3] I. Janic and M. Kakas, J. Mol. Struct. 114 (1984) 249.
[4] S. Higuchi, H. Tsuyama, S. Tanaka and H. Kamada, Spectrochım. Acta A 30 (1974) 463.

[5] C.W.N. Cumper and A.P. Thurston, J. Chem. Soc. B (1971) 422.

[6] A.N. Sobolev, V.K. Belsky, I.P. Romm. N.Y. Chernıkova and E.N. Guryanova, Acta Cryst. C 41 (1985) 967.

[7] C.B. Duke, J.W.-P Lin, A. Paton, W.R. Salaneck and K.L. Yip, Chem. Phys. Letters 61 (1979) 402.

[8] E. Shalev, N. Ben-Horın, U. Even and J. Jortner, J. Chem. Phys. 95 (1991) 3147, and references therein.

[9] Th Troxler and S. Leutwyler, J. Chem. Phys. 95 (1991) 4010 , and references therein.

[10] K. Rademann, B. Brutschy and H. Baumgärtel, Chem. Phys. 80 (1983) 129.

[11] P.D. Dao, S. Morgan and A.W. Castleman Jr., Chem. Phys. Letters 111 (1984) 38.

[12] P.D. Dao, S. Morgan and A.W. Castleman Jr., Chem. Phys. Letters 113 (1985) 219.

[13] E.J. Bieske, A.S. Uichanco, M.W. Raınbırd and A.E.W. Knıght, J. Chem. Phys. 94 ( 1991 ) 7029.

[14] P. Hermine, P. Parneix, B. Coutant, F.G. Amar and Ph. Brechıgnac, Z. Physik D, in press.

[15] G. Meijer, M.S. de Vries, H.E. Hunziker and H.R.Wendt, Appl. Phys. B 51 (1990) 395.

[16] N. Mikamı, A. Hıraya, I. Fujıwara and M. Ito, Chem. Phys. Letters 74 (1980) 531.

[17] R.A. Holroyd, J.M. Preses, E.H. Böttcher and W.F. Schmıdt, J. Phys. Chem. 88 (1984) 744.

[18] M.A. Duncan, T.G. Dietz and R.E.Smalley, J. Chem. Phys. 75 (1981) 2118.

[19] G. Meijer, M.S. de Vries, H.E. Hunziker and H.R. Wendt, J. Chem. Phys. 92 (1990) 7625

[20] E.J. Bieske, M.W. Rainbird and A.E.W. Knight, J. Chem. Phys. 90 (1989) 2068.

[21] V. Beushausen, PhD Thesıs, Unıversity of Göttingen, Germany (1990).

[22] W.A. Majewsk1 and W.L. Meerts, J. Mol. Spectry. 104 (1984) 271.

[23] W.L. Meerts, W.A. Majewski and W.M. van Herpen, Can. J.Phys. 62 (1984) 1293.

[24] W.M. van Herpen, W.L. Meerts and A. Dymanus, J. Chem. Phys. 87 (1987) 182.

[25] I. Pacansky, manuscript in preparation.

[26] M.J. Ondrechen, Z. Berkovitch-Yellın and J. Jortner, J. Am. Chem. Soc. 103 (1981) 6586.

[27] A.T. Amos, T.F. Palmer, A. Walters and B.L. Burrows, Chem. Phys. Letters 172 (1990) 503. 